CERN-TH/97-315

hep-ph/9711391

November 1997

\title{
Asymptotic expansion of Feynman integrals near threshold
}

\author{
M. BENEKE \\ Theory Division, CERN, CH-1211 Geneva 23 \\ V.A. SMIRNOV \\ Nuclear Physics Institute, Moscow State University, \\ 119889 Moscow, Russia
}

\begin{abstract}
We present general prescriptions for the asymptotic expansion of massive multiloop Feynman integrals near threshold. As in the case of previously known prescriptions for various limits of momenta and masses, the terms of the threshold expansion are associated with subgraphs of a given graph and are explicitly written through Taylor expansions of the corresponding integrands in certain sets of parameters. They are manifestly homogeneous in the threshold expansion parameter, so that the calculation of the given Feynman integral near the threshold reduces to the calculation of integrals of a much simpler type. The general method is illustrated by two-loop two-point and three-point diagrams. We discuss the use of the threshold expansion for problems of physical interest, such as the next-tonext-to-leading order heavy quark production cross sections close to threshold and matching calculations and power counting in non-relativistic effective theories.
\end{abstract}




\section{Introduction}

Many interesting processes in particle physics, in particular those in which heavy quarks participate, involve more than one mass scale. Such processes are notoriously difficult to calculate in perturbation theory beyond the one-loop level. To proceed one has to resort to approximations, either numerical or analytical. Among the latter, asymptotic expansions in certain ratios of mass scales appear most promising, because the analytic complexity is substituted by the algebraic complexity associated with obtaining a large number of terms in the expansion, which, however, can be delegated to a computer. To achieve this goal, the integrals that appear in the calculation of any given term in the expansion should (of course) be simpler than the original Feynman diagram. In particular, this means that the expansion should be manifestly homogeneous, that is, every integral that appears in the construction should contribute only to a single power in the expansion parameter.

Let us consider, for the purpose of discussion, a quantity that depends on a single kinematic invariant $q^{2}$ and a particle mass $m$, such as the two-point functions of heavy quark currents, or the production cross section for a pair of heavy quarks. There exist general explicit prescriptions [1,2, 2,3] (see 《4 for brief reviews) to obtain the asymptotic expansion of these (and other) quantities as $q^{2} \rightarrow \infty$ or $m \rightarrow \infty$. In this paper we propose a prescription to obtain the asymptotic expansion close to threshold, that is, as $q^{2} \rightarrow$ $4 m^{2}$. This limit has not been explored systematically yet, although it is of considerable interest for a field theoretical description of non-relativistic systems. We illustrate our method through examples in Sects. 2 and 4. In these examples we consider only scalar propagators. The extension to fermion and gauge field propagators complicates the numerators of the integrals, but is straightforward methodically. The general structure of the expansion, which we formalize in Sect. 3, follows the same strategy used to obtain the the expansions in limits of large/small momenta and masses, although the threshold expansion is quite different, in particular because we have to deal with three different scales near threshold. The method obviously generalizes to the threshold production of two unequal-mass particles and in fact to any particle threshold, when some massive particles are slow, although we do not treat these cases in this paper. Physically, the mathematical problem of constructing an asymptotic expansion is closely related to the notion of effective field theories. For example, the homogeneity property mentioned above translates into the property of manifest power counting for the effective Lagrangian. The threshold expansion provides some insight into how to construct a non-relativistic effective field theory within dimensional regularization, an issue that has received some attention recently. We discuss this point, together with other conclusions, in Sect. 5.

Recently Tkachov has also discussed the possibility of performing expansions near threshold [5]. However, his prescription has been applied in [5] only to the discontinu-

ity of the 1-loop 2-point diagram with two masses. The non-trivial interplay of several small scales that characterize the threshold region is seen only in more complicated loop integrals. 




Figure 1: One-loop vertex integral. Solid (wavy) lines denote massive (massless) propagators.

\section{Heuristic motivation and examples}

A typical explicit formula for the asymptotic expansion of a given Feynman integral $F_{\Gamma}$ (corresponding to a graph $\Gamma$ ) in a given limit looks like

$$
F_{\Gamma} \sim \sum_{\gamma} \mathcal{M}_{\gamma} F_{\Gamma}
$$

where the sum extends over a certain subset of subgraphs of the graph, and the operators $\mathcal{M}_{\gamma}$ perform Taylor expansions in the variables that are small in $\gamma$. To arrive at this result, one can use the following heuristic procedure: 1. Determine the large and small scales in the problem. 2. Introduce factorization scales $\mu_{i}$ and divide the loop integration domain into regions in which each loop momentum is considered to be of the order of one of the scales in the problem. 3. Perform, in every given region, a Taylor expansion in the parameters, which are small in the given region. 4. After expansion, ignore all factorization scales and integrate over the entire loop integration domain in every region.

One can easily reproduce the general formulae and combinatorical structure of the large-mass and large-momentum expansion in this way. The non-trivial point to justify is 4., which also guarantees the homogeneity of the expansion formula. In order for 4 . to be valid it is essential to use dimensional (or analytic) regularization for the Feynman integral $F_{\Gamma}$, even if $F_{\Gamma}$ is finite in four dimensions. Loosely speaking, 4. follows in dimensional regularization from the property that all integrals without scale vanish. For off-shell limits of Feynman diagrams, the above procedure can indeed be justified [2] in terms of the $R^{*}[6]$ and $R^{-1}$-operations. In this section we use these heuristic rules to treat two one-loop and one two-loop example and demonstrate that in each case the result agrees with the expansion of the exact result.

Example 1. As our first example we consider a one-loop vertex integral with two equal and one zero mass, which appears for example in the form factor for $\gamma^{*} \rightarrow \bar{Q}\left(p_{1}\right) Q\left(p_{2}\right)$ with quarks $Q$ of mass $m$, see Fig. 1. In the following we will also refer to massive lines as quarks and massless lines as gluons in general. We take $p_{1}^{2}=p_{2}^{2}=m^{2}$ and define $q=p_{1}+p_{2}$ and the relative momentum $p=\left(p_{1}-p_{2}\right) / 2$. The threshold region is 
characterized by

$$
y \equiv m^{2}-\frac{q^{2}}{4}=p^{2} \ll q^{2} .
$$

We also define $\hat{y}=y / q^{2}$ as the dimensionless parameter of the threshold expansion. The parameter $\hat{y}$ is related to a more standard parameter $\beta=\left(1-4 m^{2} / q^{2}\right)^{1 / 2}$ through $\beta=\sqrt{-4 \hat{y}}$. Above threshold $\hat{y}<0$. It is useful to choose a frame in which $q=\left(q_{0}, \overrightarrow{0}\right)$, $p_{1}=\left(p_{0}, \vec{p}\right), p_{2}=\left(p_{0},-\vec{p}\right)$ and $p=(0, \vec{p})$. In this frame, the two massive particles move slowly and can be considered as non-relativistic. The following three scales are relevant to the threshold kinematics: $q \equiv \sqrt{q^{2}} \sim 2 m$, the centre-of-mass energy, $\sqrt{-y}=|\vec{p}|$, the relative momentum of the quarks and $y / q \sim\left(p_{1,2}\right)_{0}-m$, the (non-relativistic) energy of the quarks. Accordingly, the loop momentum can be either

$$
\begin{aligned}
\operatorname{hard}(\mathrm{h}): & k_{0} \sim q, \vec{k} \sim q, \\
\text { or small }(\mathrm{sm}): & k_{0} \lesssim \sqrt{y}, \vec{k} \lesssim \sqrt{y} .
\end{aligned}
$$

Because there are two small scales, when the loop momentum is small, it can be either

$$
\begin{aligned}
\text { potential (p): } & k_{0} \sim y / q, \vec{k} \sim \sqrt{y}, \\
\text { soft (s): } & k_{0} \sim \sqrt{y}, \vec{k} \sim \sqrt{y}, \\
\text { or ultrasoft (us): } & k_{0} \sim y / q, \vec{k} \sim y / q .
\end{aligned}
$$

The terminology implies a 'canonical' routing of the large external momentum $q$ through the massive lines of the graph only. For example, in Fig. 1, one should assign the momentum $k \pm q / 2$ to the massive lines. Other routings are possible, but scaling arguments become less transparent then. The distinction between potential, soft and ultrasoft loop momentum is made only after, in the small momentum region, the integration over the zero-component of the loop momentum has been carried out by picking up the residues of the poles in $k_{0}$. The potential region is then associated with quark propagator poles and $k_{0} \ll|\vec{k}|$, because the massive particles are non-relativistic. The soft and ultrasoft regions arise from the gluon propagator poles. We shall see that in general (but not in this example) the characteristic momentum of on-shell massless particles in the small momentum region can be either $\sqrt{y} \sim m v$ or $y / q \sim m v^{2}$, where $v$ is the relative velocity of the two external quarks. (When the momentum of a massless line is in the potential region, the massless particle is off-shell by an amount of order $y$.) With these preliminaries the scalar integral of Fig. 11 is given by

$$
I_{1} \equiv \int \frac{[d k]}{\left(k^{2}+q \cdot k-y\right)\left(k^{2}-q \cdot k-y\right)(k-p)^{2}},
$$

using the kinematic variables introduced above. The standard $+i 0$-prescriptions are implicitly understood in the propagators and the integration measure is defined by $[d k] \equiv$ $e^{\epsilon \gamma_{E}} d^{d} k /\left(i \pi^{d / 2}\right)$ with $d=4-2 \epsilon$ and $\gamma_{E}=0.577216 \ldots$ The renormalization scale of dimensional regularization is set to 1 . 
When the loop momentum $k$ is hard, the integrand is expanded in $y$ and $p$. (Because $p \cdot q=0$, terms odd in $p$ vanish and the expansion produces only powers of $\hat{y}$.) The leading term is

$$
I_{1}^{h}=\int \frac{[d k]}{k^{2}\left(k^{2}+q \cdot k\right)\left(k^{2}-q \cdot k\right)}=e^{\epsilon \gamma_{E}}\left(\frac{4}{q^{2}}\right)^{1+\epsilon}\left(-\frac{1}{2}\right) \frac{\Gamma(\epsilon)}{1+2 \epsilon} .
$$

Higher-order terms in $y$ are calculated easily. The integral is evaluated most directly by reducing the number of propagators to two through partial fractioning. If one does not use partial fractions, the calculation of the integral with Feynman parameters leads to singularities inside the Feynman parameter integration domain when $d=4$, which indicate the presence of the Coulomb singularity in $I_{1}$ as $y \rightarrow 0$. This singularity is regulated by dimensional regularization as conventional ultraviolet and infrared singularities are, and $I_{1}^{h}$ is well-defined.

The contribution from the hard region corresponds to the 'naive' Taylor expansion of the integrand in $y$ and $p$. However, the integral receives an important (in fact dominant) contribution from the small loop-momentum region, where (as we will verify shortly) the spatial loop momentum is of order $\sqrt{y}$. Inspecting the quark propagator, we see that it can now be expanded in $k_{0}^{2}$ :

$$
\frac{1}{k_{0}^{2}-\vec{k}^{2}+q_{0} k_{0}-y}=\sum_{n=0}^{\infty} \frac{\left(-k_{0}^{2}\right)^{n}}{\left(-\vec{k}^{2}+q_{0} k_{0}-y\right)^{n+1}} .
$$

The leading contribution to $I_{1}$ from the small momentum region is then

$$
I_{1}^{s m}=\int \frac{[d k]}{\left(-\vec{k}^{2}+q_{0} k_{0}-y\right)\left(-\vec{k}^{2}-q_{0} k_{0}-y\right)\left(k_{0}^{2}-(\vec{k}-\vec{p})^{2}\right)} .
$$

We now perform the integration over $k_{0}$ by closing the contour in the upper complex $k_{0}$-plane.

Let us consider first the contribution from the pole of the massive ('quark') propagator at $k_{0}=-\left(\vec{k}^{2}+y\right) / q_{0}+i 0$. Since $|\vec{k}| \ll q$ by assumption, we have $k_{0} \ll|\vec{k}|$. As a consequence we might have expanded the massless propagator in (\$) in $k_{0}^{2}$ before picking up the residue from this pole. After $k_{0}$-integration one obtains a two-point function with one mass $y$ in $d-1$ dimensions with the result

$$
I_{1}^{p}=\frac{(-1)}{q} e^{\epsilon \gamma_{E}} \int \frac{d^{d-1} \vec{k}}{\pi^{d / 2-1}} \frac{1}{\left(\vec{k}^{2}+y\right)(\vec{k}-\vec{p})^{2}}=e^{\epsilon \gamma_{E}} \frac{y^{-\epsilon}}{\sqrt{q^{2} y}} \frac{\sqrt{\pi} \Gamma(\epsilon+1 / 2)}{2 \epsilon} .
$$

Because the integrand contains only one scale, the loop momentum is dominated by $\vec{k} \sim \sqrt{y}$. This justifies the scaling given in (3) for potential loop momenta. Note that higher-order terms in the expansion in $k_{0}^{2}$ yield zero, because after integration over $k_{0}$ positive powers of $k_{0}$ result in massless tadpole integrals. Note also that we could have obtained (9) by expanding all terms that are small in the potential region before integration over $k_{0}$. The contribution to the threshold expansion from the potential 
region can then easily be estimated, because by construction all terms in the denominator have the same scaling in $y$. Taking into account that $[d k] \sim y^{5 / 2} / q$ for potential $k$, we obtain $I_{1}^{p} \sim 1 / \sqrt{q^{2} y}$ as expected for the Coulomb singularity and born out by (9).

Now consider the contribution from the pole of the massless propagator in (8) located at $k_{0}=-|\vec{k}|+i 0$. (We have shifted $k \rightarrow k+p$. If one does not perform this shift, the combination $k+p$ should be considered ultrasoft, which implies a cancellation between $k$ and $p$, so that the scaling rules can not be applied to $k$ in a straightforward way.) The result is

$$
I_{1}^{s / u s}=e^{\epsilon \gamma_{E}} \int \frac{d^{d-1} k}{\pi^{d / 1-2}} \frac{1}{|\vec{k}|\left[q_{0}|\vec{k}|+\left(\vec{k}^{2}+2 \vec{p} \cdot \vec{k}\right)\right]\left[q_{0}|\vec{k}|-\left(\vec{k}^{2}+2 \vec{p} \cdot \vec{k}\right)\right]} .
$$

Expanding in the small terms $\vec{k}^{2}+2 \vec{p} \cdot \vec{k}$, the integral becomes a tadpole and is zero to all orders in the expansion. Hence, there is no contribution from the gluon pole. At first sight this looks incorrect, because the integral (10) is clearly non-zero. However, the nonzero contribution can come only from the region where $\vec{k}^{2}+2 \vec{p} \cdot \vec{k}$ is comparable to $q_{0}|\vec{k}|$, which requires $k \sim q$. This contribution is already included in the hard contribution above and (10) must be expanded to avoid double-counting. Because the integral $I_{1}^{s / u s}$ vanishes, we can not decide whether the gluon was soft or ultrasoft.

There is a useful short-cut to arrive at this result. We can perform the approximations appropriate to the soft or ultrasoft region before integrating over $k_{0}$ to obtain, to leading order in this region,

$$
I_{1}^{s / u s}=\int \frac{[d k]}{\left[q_{0} k_{0}+i 0\right]\left[-q_{0} k_{0}+i 0\right] k^{2}} .
$$

This integral is ill-defined, because the poles at $k_{0}=0$ pinch the integration contour. However, since the quark propagator poles have already been taken into account through the potential region, the previous integral should be understood as the contribution from the gluon pole only. Then one immediately arrives at a vanishing tadpole integral. Since by expanding all small quantities in the denominator, all remaining terms have the same scaling in $\sqrt{y}$, we have homogeneity (manifest power counting) also for the soft and ultrasoft regions.

One can now verify that the threshold expansion reproduces the expansion of the exact one-loop result:

$$
\begin{aligned}
I_{1}= & e^{\epsilon \gamma_{E}} y^{-1-\epsilon}{ }_{2} F_{1}\left(\frac{1}{2}, 1+\epsilon, \frac{3}{2} ;-\frac{1}{4 \hat{y}}\right) \\
= & e^{\epsilon \gamma_{E}}\left(\frac{4}{q^{2}}\right)^{1+\epsilon}\left\{\frac{(4 \hat{y})^{-\epsilon}}{\sqrt{\hat{y}}} \frac{\sqrt{\pi} \Gamma(\epsilon+1 / 2)}{8 \epsilon}\right. \\
& \left.-\frac{\Gamma(\epsilon)}{2(1+2 \epsilon)} \sum_{n=0}^{\infty} \frac{\Gamma(1+\epsilon+n)}{\Gamma(1+\epsilon)} \frac{1+2 \epsilon}{1+2 \epsilon+2 n} \frac{(-4 \hat{y})^{n}}{n !}\right\} .
\end{aligned}
$$

Note that in this example, contrary to those we discuss later, the Taylor expansions of the integrands do not generate additional poles in $\epsilon$. The poles in $\epsilon$ in both the hard and 


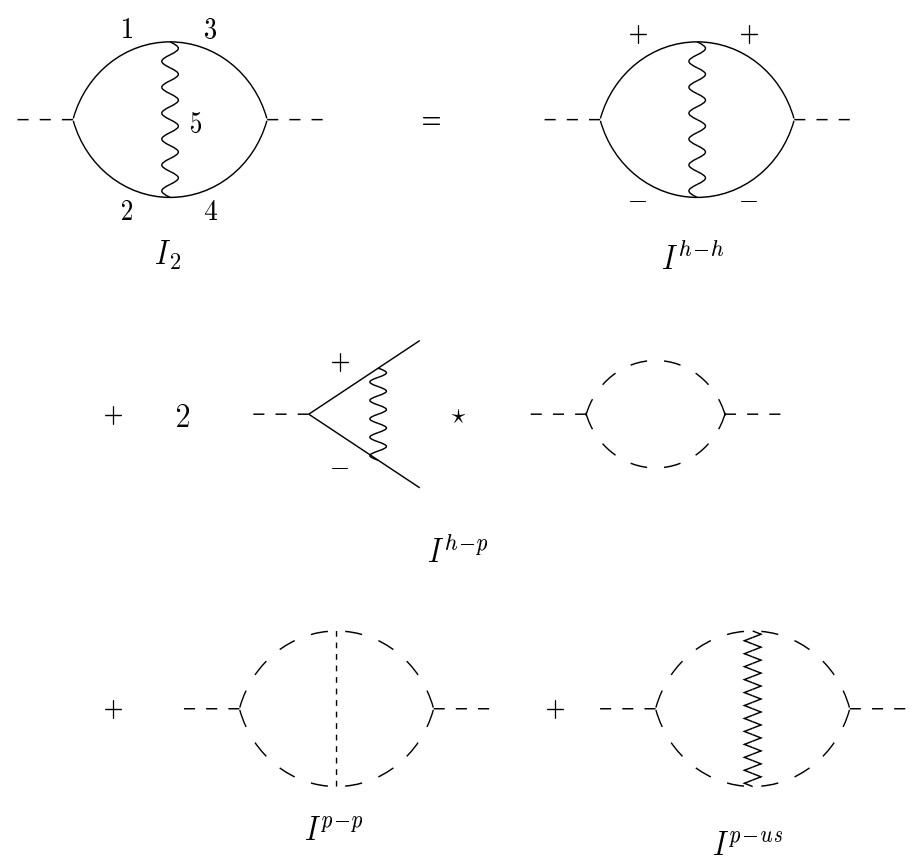

Figure 2: Diagrammatic representation of the threshold expansion for $I_{2}$. Solid lines on the left-hand side denote massive propagators, wavy lines denote massless propagators. On the right-hand side a solid line with ' $\pm^{\prime}$ denotes (hard) 'on-shell' propagators of form $1 /\left(k^{2} \pm q . k\right)$ for line momentum $k \pm q / 2$, a wavy line stands for a hard massless line. The dashed line denotes a potential massive line and the dotted line a potential massless line. The zigzagged line is an ultrasoft massless line.

the potential contribution come from the infrared pole which is present in the original integral $I_{1}$.

Example 2. We now consider the two-loop 2-point master integral. This is the first case, relevant to the two-loop corrections to heavy-quark currents, where the hard, potential and ultrasoft regions contribute in a non-trivial way to the threshold expansion. The exact result for arbitrary $\hat{y}$ serves as a check of the expansion method. The diagram, shown in Fig. 2, is given by

$$
I_{2} \equiv \int \frac{[d k][d l]}{\left(k^{2}+q \cdot k-y\right)\left(k^{2}-q \cdot k-y\right)\left(l^{2}+q \cdot l-y\right)\left(l^{2}-q \cdot l-y\right)(k-l)^{2}}
$$

and the lines in Fig. 2 are enumerated according to the order in which they appear in the denominator in (13). Expansions of this diagram in various limits of momenta and masses have been considered in [7]. The 'canonical' routing of the external momentum for this diagram assigns $\pm q / 2$ to each massive line. With this assignment, the loop 
momenta satisfy the scaling rules (3) and (4) above. For the threshold expansion of any two-loop diagram, one considers the following nine loop momentum regions: h-h: both loop momenta are hard; h-p (s, us): one loop momentum hard, the other potential (soft, ultrasoft); p-p: both loop momenta potential; p-s (us): one loop momentum potential, the other soft (ultrasoft); s-s: both loop momenta soft; s-us: one loop momenta soft, the other ultrasoft; us-us: both loop momenta ultrasoft. In general, to obtain the contributions from all possible subgraphs it is necessary to consider different assignments of loop momenta than the one chosen in (13). As we discuss shortly, some of these regions do not contribute to the case at hand. The structure of the non-vanishing terms in the expansion is shown in Fig. 2. Note that $I_{2}$ is finite in four dimensions.

When both loop momenta are of order $q$, we can expand the integrand in $y$. After partial fractioning, the resulting integrals are of type

$$
J_{ \pm}\left(a_{1}, \ldots, a_{5}\right) \equiv \int \frac{[d k][d l]}{\left[-k^{2}\right]^{a_{1}}\left[-l^{2}\right]^{a_{2}}\left[-(k-l)^{2}\right]^{a_{3}}\left[-\left(k^{2}+q \cdot k\right)\right]^{a_{4}}\left[-\left(l^{2} \pm q \cdot l\right)\right]^{a_{5}}} .
$$

The integrals of type $J_{+}$can be reduced to Gamma-functions [8] through recurrence relations derived from integration by parts [9]. The integrals of type $J_{-}$can be expressed in Gamma-functions and the integrals $J_{-}\left(0,0, a_{3}, a_{4}, a_{5}\right)$. These integrals can be reduced to $J_{-}(0,0,1,1,1)$, using a simplified version of the results of 10 . Then $J_{-}(0,0,1,1,1)$ (or a more convenient input integral) is calculated explicitly by Feynman parameters in an expansion in $\epsilon$. We obtain

$$
q^{2} I_{2}^{h-h}=\pi^{2}\left(\frac{1}{\epsilon}-2 \ln q^{2}+6 \ln 2\right)+21 \zeta(3)-4\left(8+3 \pi^{2}\right) \hat{y}+\mathcal{O}\left(\hat{y}^{2}\right) .
$$

The h-h region is already the most difficult one. When one loop momentum is hard, and the other small, one of the three subgraphs $\gamma_{1}=\{1,2,5\}, \gamma_{2}=\{3,4,5\}$ and $\gamma_{3}=\{1,2,3,4\}$ can be hard. (The numbers refer to those in Fig. 2.) The lines in the hard subgraph $\gamma_{i}$ are expanded in $y$ and $l$ (if $k$ is hard) and the quark propagators not in $\gamma_{i}$ are expanded in the zero-components squared of momenta as in (7). The resulting contribution has the factorized form

$$
F_{\Gamma / \gamma_{i}} \circ \mathcal{T}_{y} F_{\gamma_{i}}
$$

i.e. the one-loop integral $\mathcal{T}_{y} F_{\gamma_{i}}$, obtained by Taylor expansion of $F_{\gamma_{i}}$ in $y$ and $l$, generates a local vertex, which is inserted into the reduced diagram $\Gamma / \gamma_{i}$ obtained from $\Gamma$ by shrinking all lines of $\gamma_{i}$ to a point and expansion of the remaining massive propagators in the zero-components squared of the momenta.

For our example, one obtains a vanishing integral for $\Gamma / \gamma_{3}$ and equal contributions from $\gamma_{1}$ and $\gamma_{2}$. In the latter case, the non-vanishing contribution arises if the loop momentum of $\Gamma / \gamma_{1,2}$ is potential, but one obtains zero if it is soft or ultrasoft. The leading contribution from the h-p region is then (see Fig. 2)

$$
I_{2}^{h-p}=2 \int \frac{[d l]}{\left(-\vec{l}^{2}+q_{0} l_{0}-y\right)\left(-\vec{l}^{2}-q_{0} l_{0}-y\right)} \int \frac{[d k]}{k^{2}\left(k^{2}+q \cdot k\right)\left(k^{2}-q \cdot k\right)},
$$


where we expanded all terms that are small in the h-p region. It follows from powercounting that this integral contributes at order $\sqrt{\hat{y}}$. Keeping also the next term in the h-p region, we obtain

$$
q^{2} I_{2}^{h-p}=8 \pi\left(\frac{1}{\epsilon}-\ln q^{2}-\ln y\right) \sqrt{\hat{y}}-\frac{32 \pi}{3}\left(\frac{1}{\epsilon}-\ln q^{2}-\ln y+\frac{7}{3}\right) \hat{y}^{3 / 2}+\mathcal{O}\left(\hat{y}^{5 / 2}\right) .
$$

When $l$ is soft or ultrasoft, the integrand is almost the same as for (17), except that $\vec{l}^{2}$ is expanded for ultrasoft $l$ and $\vec{l}^{2}+y$ is expanded for soft $l$. In both cases, the resulting integrals vanish as already mentioned.

When both loop momenta are small, we can use (7) on all massive propagators and then take the poles in $k_{0}$ and $l_{0}$. The resulting three-dimensional integrals are

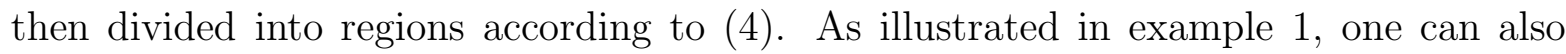
perform the appropriate approximations before integration over the zero components of loop momenta provided certain pinching quark poles in the soft or ultrasoft region are ignored as above.

When both loop momenta are potential, all propagators are expanded in their zerocomponents squared. After picking up the residues from the remaining quark poles in $k_{0}$ and $l_{0}$, the resulting $d$-1-dimensional 2-loop integral is

$$
\begin{aligned}
I_{2}^{p-p} & =-\frac{1}{q^{2}} e^{2 \epsilon \gamma_{E}} \int \frac{d^{d-1} \vec{k}}{\pi^{d / 2-1}} \frac{d^{d-1} \vec{l}}{\pi^{d / 2-1}} \frac{1}{\left(\vec{k}^{2}+y\right)\left(\vec{l}^{2}+y\right)(\vec{k}-\vec{l})^{2}} \\
& =\frac{y^{-2 \epsilon}}{q^{2}} e^{2 \epsilon \gamma_{E}} \frac{\pi \Gamma(\epsilon+1 / 2) \Gamma(\epsilon-1 / 2)}{2 \epsilon}
\end{aligned}
$$

as shown in Fig. 2. The next term and all higher order terms in the expansion in the $\mathrm{p}$-p region vanish for the same reason as in example 1 , hence

$$
q^{2} I_{2}^{p-p}=\pi^{2}\left(-\frac{1}{\epsilon}-2+4 \ln 2+2 \ln y\right) .
$$

We next consider the potential-ultrasoft region. Again we should consider all three cases, where one of the subgraphs $\gamma_{i}$ is potential and the remaining lines are ultrasoft. The only case that does not lead to vanishing scaleless integrals is potential $\gamma_{3}$, in which case the gluon line is ultrasoft. With $k$ potential and $l$ ultrasoft, the leading term is

$$
\begin{gathered}
I_{2}^{p-u s}=\int \frac{[d k][d l]}{\left(-\vec{k}^{2}+q_{0}\left(k_{0}-l_{0} / 2\right)-y\right)\left(-\vec{k}^{2}-q_{0}\left(k_{0}-l_{0} / 2\right)-y\right)} \\
\cdot \frac{1}{\left(-\vec{k}^{2}+q_{0}\left(k_{0}+l_{0} / 2\right)-y\right)\left(-\vec{k}^{2}-q_{0}\left(k_{0}+l_{0} / 2\right)-y\right)\left(l_{0}^{2}-\vec{l}^{2}\right)}
\end{gathered}
$$

where the last (massless) propagator is now not expanded in $l_{0}$. (We have chosen a more symmetric loop momentum routing than in (13).) Since $[d k] \sim y^{5 / 2} / q$ and $[d l] \sim y^{4} / q^{4}$, power-counting tells us that this contribution scales as $\sqrt{y}$. The integral is calculated by 
examining the poles in $k_{0}$ and $l_{0}$ and closing the integration contours in $k_{0}, l_{0}$ such that the number of terms is minimized. One then arrives at

$$
I_{2}^{p-u s}=\frac{2}{q^{2}} e^{2 \epsilon \gamma_{E}} \int \frac{d^{d-1} \vec{k}}{\pi^{d / 2-1}} \frac{d^{d-1} \vec{l}}{\pi^{d / 2-1}} \frac{1}{\left(\vec{k}^{2}+y\right) \overrightarrow{l^{2}}\left(2\left(\vec{k}^{2}+y\right)+q_{0}|\vec{l}|\right)} .
$$

Note that although $|\vec{k}| \gg|\vec{l}|$ in the region under consideration, the integral does not factorize in a manner comparable to (16), because of the presence of $q_{0} \gg|\vec{k}|$ in the integrand. This can be phrased as the statement that integrating out the soft momenta does not yield a local interaction vertex with respect to the ultrasoft scale. This is in contrast to integrating out the hard momentum, which does result in a local interaction with respect to the small scales, see (16), where $\mathcal{T}_{y} F_{\gamma_{i}}$ is polynomial in its (small) external momenta. Despite this fact, the above integral is effectively a succession of one-loop integrals. The result, keeping again the next term in the expansion in this region, reads

$$
q^{2} I_{2}^{p-u s}=8 \pi\left(-\frac{1}{\epsilon}-8+10 \ln 2-\ln q^{2}+3 \ln y\right)\left(\sqrt{\hat{y}}-\frac{4 \hat{y}^{3 / 2}}{3}\right)+\mathcal{O}\left(\hat{y}^{5 / 2}\right) .
$$

A technical comment on the calculation of higher-order terms in $\hat{y}$ in the potentialultrasoft region is in order. Expansion in the zero-components of the line momenta squared results in many terms, for each of which separately the integral over the semicircle at infinity does not vanish upon closing the contours in the complex plane. As a consequence one has to take care of closing the contour in the same half plane for all terms, since the contribution from the semi-circle at infinity adds to zero only in the sum.

Finally, when one momentum is potential and the other soft, we find scaleless integrals. Likewise when both momenta are either soft or ultrasoft, one obtains only vanishing integrals. This concludes the list of all relevant momentum regions.

In general, the $h-h$ and $p-p$ region contribute only to even powers of $\sqrt{\hat{y}}$ and the $\mathrm{h}-\mathrm{p}$ and p-us region contribute only to odd powers. The contribution from each region separately contains poles as $\epsilon \rightarrow 0$. The poles cancel between the $\mathrm{h}-\mathrm{h}$ and $\mathrm{p}-\mathrm{p}$ region and the $\mathrm{h}-\mathrm{p}$ and $\mathrm{p}$-us region, leaving logarithms of $\hat{y}$. The terms computed above combine to the finite threshold expansion

$$
\begin{aligned}
q^{2} I_{2}= & 2 \pi^{2} \ln (32 \hat{y})+21 \zeta(3)+16 \pi(\ln (32 \hat{y})-4) \sqrt{\hat{y}}-4\left(8+3 \pi^{2}\right) \hat{y} \\
& -\frac{64 \pi}{3}\left(\ln (32 \hat{y})-\frac{17}{6}\right) \hat{y}^{3 / 2}+\ldots
\end{aligned}
$$

The integral $I_{2}$ is known exactly for any $\hat{y}$. Using the result of 11], we have

$$
q^{2} I_{2}=F(1)+F\left(z^{2}\right)-2 F(z),
$$

where

$$
\begin{aligned}
F(z) & =6 \operatorname{Li}_{3}(z)-4 \ln z \operatorname{Li}_{2}(z)-\ln ^{2}(z) \ln (1-z), \\
z & =-\frac{1-i \sqrt{4 \hat{y}}}{1+i \sqrt{4 \hat{y}}} .
\end{aligned}
$$




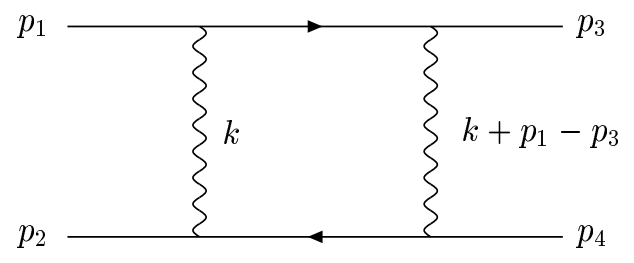

Figure 3: The box graph. Solid lines massive propagators, wavy lines massless.

Taking care of correctly continuing the logarithms in $F\left(z^{2}\right)$ to the second sheet when $\hat{y}<1 / 4$, the expansion of the exact result reproduces (24). We have verified that this agreement persists to higher orders in $\hat{y}$.

Example 3. In the previous two examples, soft (on-shell) massless lines (gluons) did not contribute to the expansion. To obtain a non-vanishing result from this region, at least two gluons have to be exchanged between the massive lines (quarks). Consider a one-loop contribution to $2 \rightarrow 2$ scattering at small relative momentum, Fig. 3, and define $p_{1 / 2}=q / 2 \pm p, p_{3 / 4}=q / 2 \pm p^{\prime}$, where $q+p_{1}+p_{2}=p_{3}+p_{4}, p=\left(p_{1}-p_{2}\right) / 2$ and $p^{\prime}=\left(p_{3}-p_{4}\right) / 2$. All external lines are on-shell, $p_{i}^{2}=m^{2}$, and with $y=m^{2}-q^{2} / 4$ as before, we have $p^{2}=p^{\prime 2}=y$. We also introduce $t=\left(p^{\prime}-p\right)^{2}$ and choose a frame where $q=\left(q_{0}, \overrightarrow{0}\right), p=(0, \vec{p})$ and $p^{\prime}=\left(0, \vec{p}^{\prime}\right)$. Then the threshold region is characterized by

$$
t \sim y \ll q^{2} .
$$

The integral represented by Fig. 3 is

$$
I_{3} \equiv \int \frac{[d k]}{\left((k+p)^{2}+q \cdot k-y\right)\left((k+p)^{2}-q \cdot k-y\right) k^{2}\left(k+p-p^{\prime}\right)^{2}} .
$$

The threshold expansion is obtained by letting the loop momentum be hard, potential, soft or ultrasoft and by expanding the integrand in all quantities that are small in the given region. In the following, we collect all terms up to order $y^{0} / q^{4}$. (Recall that the tree diagram for $2 \rightarrow 2$ scattering is $1 / t \sim 1 / y$.)

When the loop momentum is hard, the integrand is expanded in $y, p$ and $p^{\prime}$ and one obtains

$$
\left(q^{2}\right)^{2+\epsilon} I_{3}^{h}=\left(q^{2}\right)^{2+\epsilon} \int \frac{[d k]}{\left(k^{2}\right)^{2}\left(k^{2}+q \cdot k\right)\left(k^{2}-q \cdot k\right)}=-\frac{8}{3} .
$$

When the loop momentum is potential, all four propagators are expanded in $k_{0}^{2}$. This corresponds to picking up the quark propagator pole in the integration over $k_{0}$. Since the leading contribution scales as $y^{-3 / 2}$, subleading terms have to be included to achieve $\mathcal{O}\left(y^{0}\right)$ accuracy in the threshold expansion. Defining $\hat{t}=t / q^{2}$, the result is

$$
\left(q^{2}\right)^{2+\epsilon} I_{3}^{p}=\frac{\pi}{\hat{t} \sqrt{\hat{y}}}\left(\frac{1}{\epsilon}-\ln (-\hat{t})\right)+\mathcal{O}\left(\hat{y}^{1 / 2}, \hat{t}^{1 / 2}\right) .
$$


The subleading term actually vanishes in four dimensions.

Let the loop momentum be ultrasoft. It is not possible to route the external momentum $p$ or $p^{\prime}$ such that both gluon lines are ultrasoft simultaneously. Thus we get

$$
I_{3}^{u s}=\frac{1}{t} \int \frac{[d k]}{\left[q_{0} k_{0}+i 0\right]\left[-q_{0} k_{0}+i 0\right] k^{2}}=0,
$$

where the pinch at $k_{0}=0$ must be ignored, because it is taken into account by the potential region.

However, there arises a non-zero contribution from the gluon poles, when the loop momentum is soft. In this case, to leading order in the soft approximation, the integral simplifies to

$$
I_{3}^{s}=\frac{1}{q^{2}} \int \frac{[d k]}{k_{0}^{2} k^{2}\left(k+p-p^{\prime}\right)^{2}} .
$$

Closing the $k_{0}$-contour, we obtain

$$
I_{3}^{s}=\frac{1}{q^{2}} \int \frac{d^{d-1} \vec{k}}{\pi^{d / 2-1}} \frac{1}{\vec{k}^{2}-\left(\vec{k}+\vec{p}-\vec{p}^{\prime}\right)^{2}}\left(\frac{1}{|\vec{k}|^{3}}-\frac{1}{\left|\vec{k}+\vec{p}-\vec{p}^{\prime}\right|^{3}}\right) .
$$

The singularity at $\vec{k}^{2}=\left(\vec{k}+\vec{p}-\vec{p}^{\prime}\right)^{2}$ in the first factor is cancelled only in the sum of the two terms. Thus we cannot shift the momentum in the second term alone to make it equal to the first. We can calculate both terms separately, if we introduce a $+i 0$-prescription in the first factor. After this we can shift the loop momentum and obtain

$$
I_{3}^{s}=\frac{1}{q^{2}} \int \frac{d^{d-1} \vec{k}}{\pi^{d / 2-1}} \frac{1}{\left(\vec{k}^{2}\right)^{3 / 2}}\left(\frac{1}{-2 \vec{k} \cdot\left(\vec{p}-\vec{p}^{\prime}\right)+t+i 0}+\frac{1}{-2 \vec{k} \cdot\left(\vec{p}-\vec{p}^{\prime}\right)+t-i 0}\right),
$$

Now the integrals can be calculated by standard methods, taking care of the different $i 0$ prescriptions in the two terms. Again keeping the first subleading term to the threshold expansion from this region, we find

$$
\left(q^{2}\right)^{2+\epsilon} I_{3}^{s}=\frac{4}{\hat{t}}\left(-\frac{1}{\epsilon}+\ln (-\hat{t})\right)+\frac{16 \hat{y}}{3 \hat{t}}\left(\frac{1}{\epsilon}-\ln (-\hat{t})\right)+\frac{8}{3}+\mathcal{O}(\hat{y}, \hat{t}) .
$$

The contribution from the soft region is rather peculiar from the conceptual point of view. Both gluons in the box subgraph have energies of order $\sqrt{y}$, parametrically larger than the characteristic energy scale $y / q$ for the non-relativistic quarks. Hence one can imagine the exchange of the two gluons as taking place on a much shorter time scale than the one that is characteristic for a non-relativistic system. The intermediate quark pair becomes off-shell by an amount $\sqrt{q^{2} y}$ through the interaction with soft gluons, while the off-shellness of the quarks remains of order $y$ in the potential and ultrasoft regions. The soft region thus can be considered intermediate between the hard region (off-shellness of order $q^{2}$ ) and the two other small momentum regions. However, while a hard subgraph can be interpreted as a local interaction vertex, the soft box subgraph, when it appears 
as a subgraph in a 2-loop diagram gives rise to a vertex which is non-local in its external three-momenta but local in the zero-components of the external momenta as we discuss in more detail in Sects. 3 and 4. We also understand why the soft region did not appear in the previous two examples. Since an external quark pair or a quark pair that couples to the external current is off-shell by an amount of order $y$, after a quark interacts with a soft gluon which puts it off-shell by an amount of order $\sqrt{q^{2} y}$, it has to interact with at least a second soft gluon to reduce its off-shellness to $y$ again.

The threshold expansion can be compared with the very simple exact (up to terms that vanish as $\epsilon \rightarrow 0$ ) result for the box diagram [12]:

$$
\begin{aligned}
\left(q^{2}\right)^{2+\epsilon} I_{3} & =\frac{2}{\hat{t} \sqrt{\hat{y}}}\left[\frac{\pi}{2}-\arctan \sqrt{4 \hat{y}}\right]\left(\frac{1}{\epsilon}-\ln (-\hat{t})\right) \\
& =\left[\frac{\pi}{\hat{t} \sqrt{\hat{y}}}-\frac{4}{\hat{t}}+\frac{16 \hat{y}}{3 \hat{t}}+\ldots\right]\left(\frac{1}{\epsilon}-\ln (-\hat{t})\right) .
\end{aligned}
$$

The expansion in $\hat{y}$ and $\hat{t}$ agrees with the sum of the terms computed above. Note the cancellation between the hard region and the constant from the soft region, which has to repeat itself in higher orders due to the factorized $t$-dependence of the exact result.

\section{General structure of the threshold expansion}

In the previous section we have been rather explicit and worked out the threshold expansion on simple examples. In this section we abstract from these examples general prescriptions for the threshold expansion $\left(y=m^{2}-q^{2} / 4\right.$ and other small parameters such as $\hat{t}$ in example 3 tend to zero at fixed $m^{2}$ ) of an arbitrary diagram which contains two paths of lines with 'slow' particles with mass $m$ ('quarks') and some massless particles ('gluons'). (Internal loops of massive particles can be included straightforwardly: If all momenta that connect to the internal heavy quark loop are small, the integrand is expanded in external momenta over the mass and reduces to a series of local operators analogous to the Euler-Heisenberg effective Lagrangian. If large momentum flows through part of the graph, the corresponding lines are not expanded. With this in mind, the following discussion is adapted to diagrams without internal heavy quark loops.) The two paths of massive lines are assigned $q / 2$ and $-q / 2$ of the large external momentum $q$. The two paths can be either disconnected as in the box graph example or they can be joined together by a hard external vertex that ejects large momentum $q$ into the graph, such as in the two-point and three-point functions discussed in Sect. 2. We also assume a frame such that $q=\left(q_{0}, \overrightarrow{0}\right)$. The loss of explicit covariance is intrinsic to the threshold problem and, while an explicitly covariant formulation is possible, it would be exceedingly cumbersome.

We arrive at our general prescription in three steps, which correspond to dealing with the hierarchy of scales sequentially. To arrive at the first form of the expansion, we consider any loop momentum to be either large or small. However, at this step, we do not identify small momenta as potential, soft or ultrasoft. In particular, when a loop 
momentum that flows through a quark line is small, the zero-component squared of the loop momentum in the quark propagator is small, see (7). Thus, at the first step, we arrive at the following expansion

$$
F_{\Gamma}=\sum_{\gamma} \mathcal{T}_{k_{i 0}^{2}} F_{\Gamma / \gamma} \circ \mathcal{T}_{k^{\gamma}, y} F_{\gamma}
$$

where the sum is in one-particle-irreducible (1PI) subgraphs of the given graph $\Gamma$. The operator $\mathcal{T}_{k^{\gamma}, y}$ in the second factor performs the Taylor expansion of the Feynman integral $F_{\gamma}$ in $y$ and the loop momenta $k^{\gamma}$ of $\Gamma$ which are external with respect to $\gamma$. (These are the loop momenta of the reduced graph $\Gamma / \gamma$, which is obtained by shrinking all lines of $\gamma$ to a point.) The operator $\mathcal{T}_{k_{i 0}^{2}}$ in the first factor performs the Taylor expansion of the Feynman integral $F_{\Gamma / \gamma}$ associated with the reduced graph in the squares of the zerocomponents of the loop momenta that flow through the quark lines of the given paths. The above equation has the combinatorical structure of a factorized expression. It is easy to see that (38) is equivalent to constructing a non-relativistic effective Lagrangian [13]. The factors $\mathcal{T}_{k^{\gamma}, y} F_{\gamma}$ can be associated with the insertion of local operators into the diagram and they account for all hard loop momenta. The factors $\mathcal{T}_{k_{i 0}^{2}} F_{\Gamma / \gamma}$ can be associated with diagrams in the effective theory and the hard scale $q$ is not present as a scale for loop momenta in this part any more. Note that the asymptotic expansions for certain limits of on-shell integrals considered in [14] can be recast into a form similar to (38), with expansion in $k^{2}$ rather than $k_{0}^{2}$ in the small momentum part.

However, the expansion (38) is not homogeneous in the expansion parameter. The non-homogeneity arises from the small momentum parts $\mathcal{T}_{k_{i 0}^{2}} F_{\Gamma / \gamma}$, which are still nontrivial series in the threshold expansion parameter(s). To obtain the expansion in a manifestly homogeneous form we have to specify further the scale of the loop momenta of the reduced graphs $\Gamma / \gamma$ in (38). At this point we consider potential, soft and ultrasoft loop momenta with the scaling rules given in (4). Let $\hat{\Gamma}$ be a reduced graph that appears in (38). Since we have already disposed of all hard momentum regions, the 'next hardest' region is the region of soft loop momenta. Consider a soft subgraph $\gamma_{s}$ of $\hat{\Gamma}$, i.e. a 1PI subgraph of $\hat{\Gamma}$ in which all loop momenta $l^{\gamma_{s}}$ scale as $l_{0}^{\gamma_{s}} \sim \vec{l}^{\gamma_{s}} \sim \sqrt{y}$ and the external momenta $k^{\gamma_{s}}$ of $\gamma_{s}$ are either potential or ultrasoft. Then $\gamma_{s}$ is expanded in all external momenta, which are ultrasoft, and reduces to a local interaction with respect to the ultrasoft scale. For a potential external momentum, we expand only in its zero component, because the spatial component of a potential momenta is of the same order as the spatial component of a soft momentum. As a consequence, the soft subgraph is a temporally local vertex with respect to the potential scale, but it is spatially non-local, because the dependence on the three-momenta of the potential external momenta is not polynomial. Therefore, defining $\hat{F}_{\hat{\Gamma}}=\mathcal{T}_{k_{i 0}^{2}} F_{\hat{\Gamma}}$ as it appears in (38), we arrive at

$$
\hat{F}_{\hat{\Gamma}}=\sum_{\gamma_{s}} \hat{F}_{\hat{\Gamma} / \gamma_{s}} \circ \mathcal{T}_{k_{u s}^{\gamma_{s}}, k_{p, 0}^{\gamma_{s}}} \hat{F}_{\gamma_{s}}
$$

where the sum is again in all 1PI subgraphs. In addition to the Taylor expansions 
indicated explicitly, the massive propagators in $\gamma_{s}$ should be expanded according to

$$
\frac{1}{-\left(\sum_{i} \vec{l}_{\gamma_{s}}+\sum_{j} \vec{k}^{\gamma_{s}}\right)^{2} \pm q_{0}\left(\sum_{i} \vec{l}_{0}^{\gamma_{s}}+\sum_{j} \vec{k}_{0}^{\gamma_{s}}\right)-y}=\frac{1}{ \pm q_{0} \sum_{i} \vec{l}_{0}^{\gamma_{s}}}+\ldots
$$

and pinched poles are simply to be ignored, see the discussion in Sect. 2. Notice that these are static propagators rather than non-relativistic propagators. After inserting (39) in (38) we have achieved factorization of both hard and soft momenta. The factors $\mathcal{T}_{k_{u s}^{\gamma_{s}}, k_{p, 0}^{\gamma_{s}}} F_{\gamma_{s}}$ can be interpreted as an instantaneous interaction vertex, and thus as contribution to the heavy quark potential. On the other hand, since $\mathcal{T}_{k_{u s}^{\gamma_{s}}, k_{p, 0}^{\gamma_{s}}} \hat{F}_{\gamma_{s}}$ is spatially non-local, the graph $\hat{\Gamma} / \gamma_{s}$ is interpreted as deleting all lines of $\gamma_{s}$ in $\hat{\Gamma}$ and replacing them by $\mathcal{T}_{k_{u s}^{\gamma_{s}}, k_{p, 0}^{\gamma_{s}}} \hat{F}_{\gamma_{s}}$. Let us give an example. The soft part of the box graph of Sect. 2 has four potential external quark lines and we have

$$
\mathcal{T}_{k_{u s}^{\gamma_{s}}, k_{p, 0}^{\gamma_{s}}} \hat{F}_{b o x} \rightarrow \frac{c}{q_{0}^{2}} \frac{1}{\left[-\left(\vec{p}-\vec{p}^{\prime}\right)^{2}\right]^{1+\epsilon}}
$$

with a numerical constant $c$. In coordinate space, this gives rise to a non-local fourfermion operator. Due to the structure of (39), the factorization of soft regions can also be implemented as an effective Lagrangian, although it is non-local in space. In the planar two-loop three-point integral which we treat in Sect. 4, we will see how such a nonlocal operator inserted into a reduced diagram contributes to the threshold expansion of two-loop diagrams. In Sect. 4 we will also meet another non-local vertex for the interaction of two potential quark lines with one potential gluon line. One can use power counting for the soft region to determine all non-local operators which contribute to a given order in the threshold expansion.

The expansion obtained from combining (38) and (39) is still not homogeneous in the threshold expansion parameter. The inhomogeneity arises from $\hat{F}_{(\Gamma / \gamma) / \gamma_{s}}$, where hard and soft lines are deleted, but potential and ultrasoft loop momenta are still not separated. First, we note that quark lines can never be ultrasoft. Technically, this is so, because, since only $k_{0}$ can combine with a large $q_{0}$, spatial tadpoles always result, if one tries to make quark lines ultrasoft. As a consequence, one should consider all contributions in which all quark lines are potential and a (possibly empty) collection of (possibly disconnected) gluon lines is ultrasoft. When an ultrasoft gluon line with momentum $l$ connects to a quark line with loop momentum $k-l / 2$ for the incoming and $k+l / 2$ for the outgoing quark line, we can expand the quark-gluon vertex and quark propagator in $\vec{l} / \vec{k} \sim \sqrt{\hat{y}}$. This corresponds to a multipole expansion, as the wavelength of ultrasoft gluons is large as compared to separation of the quark-antiquark system in a coordinate space picture. After multipole expansion, all scales are separated and we arrive at a fully homogeneous threshold expansion.

We have arrived at this prescription in a heuristic and descriptive way. We are presently not able to give a mathematical proof of our prescriptions similar to what is available for off-shell limits [3] and leave a mathematical treatment to future publications. To complete such a proof for the threshold expansion one needs to use the 
$\alpha$-representation and a subsequent decomposition of the integration domain into appropriate sectors which should be introduced, in the language of the $\alpha$-parameters, in a way analogous to our decomposition into regions of large, potential, soft and ultrasoft momenta. In fact, a proof of the asymptotic expansions for off-shell limits [3] reduces to the analysis of the asymptotic behaviour of the remainder, which has the structure of the $R$-operation, i.e. renormalization at the diagrammatic level. Therefore this proof has much in common with standard proofs of the finiteness of the renormalized Feynman integrals.

According to the definition of the asymptotic expansion, its remainder possesses a given asymptotic behaviour (a sufficiently fast decrease) once one keeps a sufficiently large number of the terms of the expansion. As in the case of the limits for which explicit general formulae were known up to now [1, 2, 2,3, 14, it is possible to characterize the remainder of the threshold expansion in terms of the $R$-operation. Since the product of any two (or more) subtraction operators that are present in the prescriptions for the asymptotic expansions is zero, we can rewrite the sum that in the right-hand side of the expansion (1) as

$$
\sum_{\gamma} \mathcal{M}_{\gamma}=1-\mathcal{R}
$$

where the operation $\mathcal{R}$ has the structure of the usual (ultraviolet) $R$-operation and is given by the forest formula

$$
\mathcal{R}=\sum_{\mathcal{F}} \prod_{\gamma \in \mathcal{F}}\left(-\mathcal{M}_{\gamma}\right)
$$

For off-shell limits, the operator $\mathcal{M}_{\gamma}$ performs Taylor expansion of the Feynman integral $F_{\gamma}$ in its small masses and external momenta and inserts the resulting polynomial in the reduced diagram $F_{\Gamma / \gamma}$. In our case, the Taylor expansions appropriate to a given region should be implied. In (43) the sum is in forests (i.e. subsets of non-overlapping subgraphs) composed of subgraphs that are involved in the prescriptions for the asymptotic expansion in the given limit. Therefore, we can represent an initial Feynman integral as

$$
F_{\Gamma}=\sum_{\gamma} \mathcal{M}_{\gamma} F_{\Gamma}+\mathcal{R} F_{\Gamma}
$$

The remainder is constructed in such a way that it is finite (if the original diagram is finite) and possesses a desired estimate in the given limit. The order of the expansion is implicitly hidden in degrees of the subtractions operators involved. From the mathematical point of view, the subtractions performed by the operators $\mathcal{M}_{\gamma}$ corresponding to subgraphs $\gamma \neq \Gamma$ remove divergences generated by the naive expansion (i.e. for $\gamma=\Gamma$ ) of the integrand in the expansion parameter. This naive expansion in turn removes divergences that could appear due to the subtractions in the subgraphs.

Let us illustrate the above points through our examples. In example 1, we have the following expression for the remainder:

$$
\mathcal{R} I_{1} \equiv \int[d k]\left(1-\mathcal{T}_{y}^{N}\right)\left(1-\mathcal{T}_{k_{0}^{2}}^{N}\right) \frac{1}{\left(k^{2}+q \cdot k-y\right)\left(k^{2}-q \cdot k-y\right)(k-p)^{2}}
$$


Here the superscript $N$ denotes the order of the Taylor expansion. It turns out that the remainder possesses a necessary asymptotic behaviour, provided the degree of the subtraction operators is chosen sufficiently large, and does not have divergences that were not present from the very beginning. Consider first the region of small $k$. Then the operator $\mathcal{T}_{y}^{N}$ is dangerous, in the sense that it generates an infrared divergence that appears when $y \rightarrow 0$. However, the second factor $\left(1-\mathcal{T}_{k_{0}^{2}}^{N}\right)$ kills this divergence because it either provides additional factors of $k$, or explicit powers of $y$ (which effectively reduce the order of expansion in $y$ in the first factor). Similarly, in the region of large $k$, the operator $\mathcal{T}_{k_{0}^{2}}^{N}$ is dangerous because it generates positive powers of $k$. This time, the first factor $\left(1-\mathcal{T}_{y}^{N}\right)$ improves the convergence of the whole integral.

Consider example 2. As a remainder we now have $\mathcal{R} F_{\Gamma}$ where

$$
\mathcal{R}=\left(1-\mathcal{M}_{\Gamma}\right)\left(1-\mathcal{M}_{h-p}-\mathcal{M}_{p-h}\right)\left(1-\mathcal{M}_{p-p}-\mathcal{M}_{p-u s}\right)
$$

The operators $\mathcal{M}_{h-p}, \mathcal{M}_{p-h}, \mathcal{M}_{p-p}$ and $\mathcal{M}_{p-u s}$ have to remove infrared divergences generated by the action of the operator $\mathcal{M}_{\Gamma}=\mathcal{M}_{h-h}=\mathcal{T}_{y}$. Consider, for example, the region where $k$ is small while $l$ is non-zero. Then one can pick up the bracket $\left(1-\mathcal{M}_{h-p}\right)$ which makes the term with $\mathcal{M}_{\Gamma} F_{\Gamma}$ convergent. In the region where both $k$ and $l$ are small, this is the operator $\mathcal{M}_{p-p}$ that provides convergence that could be spoiled by the operator $\mathcal{M}_{\Gamma}$, and the operator $\mathcal{M}_{p-u s}$ that kills the divergence at $k, l \rightarrow 0$ caused by $\mathcal{M}_{h-p}$ and $\mathcal{M}_{p-h}$.

As in the case of known limits of momenta and masses, we have, in the right handside of (45) and (46), and (38) and (39), an interplay between ultraviolet and infrared divergences which manifest themselves as poles in $\epsilon$. However, the naive expansion (in $y$ ) does not always generate additional poles — see, e.g., example 1. Still the naive Taylor expansion part taken alone does not provide a correct answer to the problem and should be accompanied by extra contributions.

\section{Two-loop vertex integrals}

In this section we discuss and present results on the threshold expansion of two-loop 3-point functions, which, to our knowledge can not be obtained from expansion of an exact result. The three-point functions are also the non-trivial building blocks for many physical applications. Here we consider the abelian planar and non-abelian graphs in Fig. tha and b. The non-planar graphs (Fig. Ac) will be discussed together with first applications in [15].

The abelian planar graph. Define the one-loop subgraphs $\gamma_{1}=\{1,3,5\}, \gamma_{2}=$ $\{2,4,5,6\}$ and $\gamma_{3}=\{1,2,3,4,6\}$. (The numbering of lines refers to Fig. 4a.) The unexpanded integral is given by

$$
I_{\mathrm{PL}} \equiv \int \frac{[d k][d l]}{\left(k^{2}+q \cdot k-y\right)\left(k^{2}-q \cdot k-y\right)\left(l^{2}+q \cdot l-y\right)\left(l^{2}-q \cdot l-y\right)(k-l)^{2}(l-p)^{2}},
$$




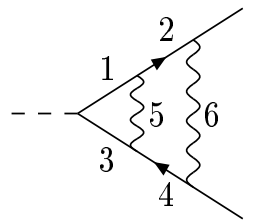

(a)

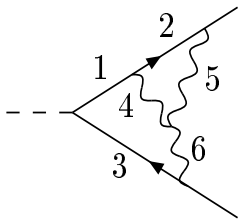

(b)

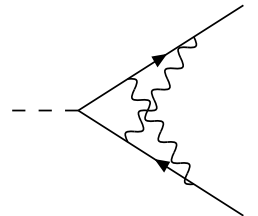

(c)

Figure 4: Examples of two-loop three-point graphs. Solid (wavy) lines denote massive (massless) lines.

where the kinematic variables are defined as in example 1 above. Inspecting all regions that contribute to the threshold expansion, we find only six of them non-vanishing. Together with their leading contribution (up to logarithms of $\hat{y}$ ) to $q^{4} I_{\mathrm{PL}}$, they are:

$$
\begin{aligned}
& \text { h-h: } 1 \\
& \text { h-p, with } \gamma_{1} \text { hard: } 1 / \sqrt{\hat{y}} \\
& \text { h-p, with } \gamma_{2} \text { hard: } \sqrt{\hat{y}} \\
& \text { p-p: } 1 / \hat{y} \\
& \text { p-s, with } \gamma_{2} \text { soft: } 1 / \sqrt{\hat{y}} \\
& \text { p-us, with } \gamma_{3} \text { potential: } 1 / \sqrt{\hat{y}} .
\end{aligned}
$$

Let us emphasize that the scalings given here follow from power counting alone, because in each region the integrand is homogeneous in the expansion parameter.

The calculation of the various contributions is straightforward in the sense that all non-trivial aspects of the calculation have been met in the examples discussed in detail in Sect. 2. For instance, after partial fractioning, the integrals needed to evaluate the h-h region reduce to $J_{ \pm}$of (14) (with additional scalar products in the numerator) and the complexity of integrals is essentially the same as for the 2-point function of example 2. The leading contribution close to threshold comes from the p-p region and corresponds to the double Coulomb exchange. Let us consider in more detail the fifth contribution, when the box subgraph is soft and loop momentum $k$ that flows through lines 1,3 and 5 is potential. First, when we take the integral over the other loop momentum $l$ first, the result is identical to the soft contribution to the box subgraph in example 3 of Sect. 2, except that the difference of external relative momenta $p-p^{\prime}$ is replaced by the difference of the external momenta of the box subgraph $p+k-p=k$. The momentum dependence of the box subgraph is $1 /\left(\vec{k}^{2}\right)^{1+\epsilon}$, which can be interpreted as a non-local vertex. Second, 
using $p \cdot q=0$, it is easy to see that in every region the expansion parameter is $\hat{y}$, except when one momentum is soft, in which case the expansion parameter is $\sqrt{\hat{y}}$. However, for the box subgraph the symmetry between the upper and lower quark line eliminates all odd terms in $\sqrt{\hat{y}}$ and subleading contributions are suppressed by integer powers of $\hat{y}$.

The integral $I_{\mathrm{PL}}$ is infrared divergent. Collecting all terms up to order $\hat{y}^{0}$ close to threshold, we obtain

$$
\begin{aligned}
& \left(\frac{q^{2}}{4}\right)^{2+2 \epsilon} I_{\mathrm{PL}}=\frac{1}{\epsilon^{2}}\left[\frac{\pi^{2}}{128 \hat{y}}-\frac{\pi}{16 \sqrt{\hat{y}}}+\frac{1}{8}\right]+\frac{1}{\epsilon}\left[-\frac{\pi^{2}}{64 \hat{y}} \ln (16 \hat{y})+\frac{\pi}{8 \sqrt{\hat{y}}}(2-\ln 2)-\frac{1}{4}\right] \\
& +\frac{\pi^{2}}{64 \hat{y}}\left(\ln ^{2}(16 \hat{y})+\frac{7 \pi^{2}}{12}\right)+\frac{\pi}{8 \sqrt{\hat{y}}}\left(\ln ^{2}(16 \hat{y})+(3 \ln 2-4) \ln (16 \hat{y})+\ln ^{2} 2-2 \ln 2+\frac{\pi^{2}}{4}\right) \\
& -\frac{29 \pi^{2}}{48}-\frac{3}{2}+\mathcal{O}\left(\hat{y}^{1 / 2}\right) .
\end{aligned}
$$

The calculation of higher-order terms can be automated on a computer. As a check of this result, we have calculated the exact double pole part of the integral,

$$
\left(\frac{q^{2}}{4}\right)^{2+2 \epsilon} I_{\mathrm{PL} \mid \frac{1}{\epsilon^{2}}}=\frac{1}{32 \epsilon^{2} \hat{y}}\left(\frac{\pi}{2}-\arctan \sqrt{4 \hat{y}}\right)^{2}
$$

and the first three terms in the expansion agree with the double pole part of (49).

The non-abelian graph. For the diagram of Fig. $4 \mathrm{~b}$, we define the one-loop subgraphs $\gamma_{1}=\{2,4,5\}, \gamma_{2}=\{1,3,4,6\}$ and $\gamma_{3}=\{1,2,3,5,6\}$. The unexpanded integral is given by

$$
I_{\mathrm{NA}} \equiv \int \frac{[d k][d l]}{\left(k^{2}+q \cdot k-y\right)\left(k^{2}-q \cdot k-y\right)\left(l^{2}+q \cdot l-y\right)(k-l)^{2}(k-p)^{2}(l-p)^{2}} .
$$

Inspecting all regions that contribute to the threshold expansion, we find only four of them non-vanishing. Together with their leading contribution (up to logarithms of $\hat{y}$ ) to $q^{4} I_{\mathrm{NA}}$, they are:

h-h: 1

$$
\begin{array}{r}
\text { h-p, with } \gamma_{1} \text { hard: } 1 / \sqrt{\hat{y}} \\
\text { p-s, with } \gamma_{1} \text { soft: } 1 / \hat{y} \\
\text { p-us, with } \gamma_{3} \text { potential: } 1 / \sqrt{\hat{y}} .
\end{array}
$$

The p-p region that scales as $1 / \hat{y}$ does not contribute here, because the poles in $l_{0}$ lie on one side of the real axis only. Hence, the $l_{0}$-integral vanishes. The potential-soft region is algebraically most complicated. Since the soft vertex subgraph $\gamma_{1}$ is less symmetric than the box subgraph of the abelian planar diagram, the expansion in this region runs 
in $\sqrt{\hat{y}}$ and it is necessary to compute the first two subleading terms in the expansion in this region to obtain an accuracy $\mathcal{O}\left(\hat{y}^{0}\right)$ at threshold. The integral $I_{\mathrm{NA}}$ is infrared divergent. Collecting all terms up to order $\hat{y}^{0}$ close to threshold, we obtain

$$
\begin{aligned}
& \left(\frac{q^{2}}{4}\right)^{2+2 \epsilon} I_{\mathrm{NA}}=\frac{1}{\epsilon^{2}}\left[-\frac{\pi}{32 \sqrt{\hat{y}}}+\frac{1}{8}\right]+\frac{1}{\epsilon}\left[\frac{\pi^{2}}{64 \hat{y}}-\frac{\pi^{2}}{16}-\frac{1}{4}\right]-\frac{\pi^{2}}{32 \hat{y}}(\ln (16 \hat{y})+2) \\
& +\frac{\pi}{32 \sqrt{\hat{y}}}\left(\ln ^{2}(16 \hat{y})+8 \ln (32 \hat{y})+\frac{5 \pi^{2}}{6}\right)+\frac{\pi^{2}}{8} \ln (16 \hat{y})-\frac{23 \pi^{2}}{48}-\frac{3}{2}+\mathcal{O}\left(\hat{y}^{1 / 2}\right) .
\end{aligned}
$$

As a check of this result, we have calculated the exact double pole part of the integral,

$$
\left(\frac{q^{2}}{4}\right)^{2+2 \epsilon} I_{\mathrm{NA} \mid \frac{1}{\epsilon^{2}}}=-\frac{1}{16 \epsilon^{2} \sqrt{\hat{y}}} \frac{1}{1+4 \hat{y}}\left(\frac{\pi}{2}-\arctan \sqrt{4 \hat{y}}\right),
$$

and the first two terms in the expansion agree with the double pole part of (53).

We close this section with some observations on the non-planar diagram, Fig. \&c, postponing a detailed discussion to [15]. The non-vanishing regions are the same as for the non-abelian diagram, with the difference that the hard-potential contribution scales as $\sqrt{\hat{y}}$ in leading order and the potential-soft region as $1 / \sqrt{\hat{y}}$ as for the planar abelian diagram. (The p-p region vanishes again, because all poles in one of the zero-component integrations lie on one side of the real axis.) Any integral that contributes to the h-h region can be reduced to either $J_{ \pm}$or

$$
\begin{aligned}
& L_{ \pm}\left(a_{1}, \ldots, a_{5}\right) \equiv \\
& \quad \int \frac{[d k][d l]}{\left[-k^{2}\right]^{a_{1}}\left[-l^{2}\right]^{a_{2}}\left[-\left((k+l)^{2}+q \cdot(k+l)\right)\right]^{a_{3}}\left[-\left(k^{2}+q \cdot k\right)\right]^{a_{4}}\left[-\left(l^{2} \pm q \cdot l\right)\right]^{a_{5}}} .
\end{aligned}
$$

With integration by parts we can reduce these integrals first to $L_{+}\left(0,0, a_{3}, a_{4}, a_{5}\right)=$ $L_{-}\left(0,0, a_{3}, a_{4}, a_{5}\right)$ and then to a single integral such as $L_{+}(0,0,2,2,1)$. (The algorithm for $L_{+}$has been used before in [8,16], although no details have been given there.) Furthermore, the hard parts of all possible 2-loop 3-point graphs relevant to QCD can be reduced to $J_{ \pm}$or $L_{ \pm}$and, consequently, can be solved in the threshold expansion.

\section{Discussion}

The threshold expansion, which we proposed and illustrated in this paper, could be applied to two sets of problems. The first set consists of problems where it is desirable to have as many terms in the expansion as can possibly be calculated. The second consists of problems where the nature of the problem specifies that only a limited number of terms are needed.

The cross section for the production of heavy quarks in $e^{+} e^{-}$annihilation $\sigma_{e^{+} e^{-}}\left(q^{2}, m\right)$ at intermediate energies $\sqrt{q^{2}}$ is an important example of the first kind. Presently available techniques do not permit an analytic calculation of $\sigma_{e^{+} e^{-}}\left(q^{2}, m\right)$ at order $\alpha_{s}^{2}$. In [17] 
the large-momentum and large-mass expansion together with convergence accelerating methods have been used to approximate $\sigma_{e^{+} e^{-}}\left(q^{2}, m\right)$ to high accuracy from expansions around $q^{2}=0$ and $q^{2}=\infty$. It is exactly the threshold point $q^{2}=4 m^{2}$ that is most critical in this approach. The threshold expansion, when computed to high order, provides us with an intermediate point, the expansion around which can probably be smoothly joined with the large-momentum and large-mass expansion in order to obtain an accurate result for $\sigma_{e^{+} e^{-}}\left(q^{2}, m\right)$ for any $q^{2}$ except very close to threshold, where a resummation of all corrections of form $\alpha_{s}^{0,1,2}\left(\alpha_{s} / \sqrt{\hat{y}}\right)^{n}$ is necessary. (This resummation belongs to the second set of problems.) The analysis of the 2-loop 2-point function in Sect. 2 suggests that the threshold expansion for $\sigma_{e^{+} e^{-}}\left(q^{2}, m\right)$ converges when $q^{2} \in\left[0,8 m^{2}\right]$. Therefore we expect that a reasonably accurate approximation to $\sigma_{e^{+} e^{-}}\left(q^{2}, m\right)$ could be obtained for $q^{2}<6 m^{2}$ starting from the threshold expansion.

In this paper we have treated only the threshold expansion of loop integrals, but not of phase space integrals for real radiation. However, as in [17] it seems advantageous to consider the three-loop vacuum polarisation of heavy quarks in the threshold expansion in order to avoid the separate calculation of virtual corrections and real radiation. Since $\sigma_{e^{+} e^{-}}\left(q^{2}, m\right)$ requires only the imaginary part of the two-point function, one can take advantage of this fact from the beginning. For example, the region where all three loop momenta are hard, which would be very difficult to calculate, is analytic in $\hat{y}$ and therefore does not contribute to the imaginary part. Other contributions reduce to twoloop diagrams (such as those of Sect. 4) or simple three-loop diagrams.

The second set of problems involves 'matching calculations'. In this case the quark mass $m$ is a large scale and the observable in question should be factorized into a shortdistance contribution from the scale $m$ and a long-distance contribution which can be either perturbative or non-perturbative. Examples of this kind include bound state calculations in QED, quarkonium systems in QCD, but also the above-mentioned resummation of 'Coulomb-enhanced' corrections in heavy quark production close to threshold. In order to obtain the matching coefficient for a given operator, a suitable (typically on-shell) Green function has to be expanded close to threshold to a certain order, which is determined by the structure and dimension of the operator. As already mentioned in Sect. 3, the combinatorical structure of the separation of large (hard) and small momenta in the threshold expansion, which is manifest in (38) for a given diagram $\Gamma$, translates into the statement of factorization

$$
G\left(m, p_{i}\right)=\sum_{i} C_{i}(m) G_{O_{i}}\left(p_{i} / m\right)
$$

for the Green function $G$ to which $\Gamma$ contributes. Here $O_{i}$ is a local operator, inserted into $G$, and $C_{i}$ is its short-distance coefficient. This construction is completely equivalent to the construction of a non-relativistic effective theory [13] in dimensional regularization. In particular (38) leads to the factorization prescription that for any diagram $\Gamma$ its contribution to the short-distance coefficient(s) ('hard part') is given by selecting only $\gamma=\Gamma$ in the sum over all subgraphs:

$$
F_{\Gamma \mid \text { hard }}=\mathcal{T}_{y} F_{\Gamma}
$$


That is, the hard part is given by the 'naive' Taylor expansion of $\Gamma$. Note that this is not identical to dropping all singular terms at threshold. For example, the abelian planar 3-point integral in Sect. 4 contains terms proportional to $\hat{y}^{0}$ both from the h-h and the p-p region. The contribution from the second region should not be considered as part of the short-distance coefficient. The non-relativistic approximation to the quark propagator in (7) does not coincide exactly with the standard convention. Let the gluon line in the triangle graph of Fig. 1 have momentum $k$ and the upper quark line have momentum $k+p_{1}$. In the standard convention one would introduce an external momentum $\tilde{p}=\left(\tilde{p}_{0}, \vec{p}\right)$ with the non-relativistic on-shell condition $\tilde{p}_{0}=\vec{p}^{2} /(2 m)$. The non-relativistic propagator is then

$$
\frac{1}{2 m} \frac{1}{\tilde{p}_{0}+k_{0}-\frac{(\vec{p}+\vec{k})^{2}}{2 m}}=\frac{1}{-(\vec{k}+\vec{p})^{2}+q_{0} k_{0}-y+\delta},
$$

where the difference to the approximation used in this paper is $\delta=\left(q_{0} / 2-m\right) k_{0}=$ $\left(\sqrt{m^{2}-y}-m\right) k_{0}$, which is smaller than all other terms in the denominator and can be expanded. Thus our small-momentum approximation is equivalent to a reparametrization of NRQED/NRQCD, where the large part of the massive line momentum is taken to be $q / 2$ rather than $m$.

Once the hard momenta are factorized ('integrated out'), an interesting aspect of non-relativistic effective theory is that it does not lead to manifest power counting. This means that the matrix elements of operators are series in the small parameters, rather than being homogeneous, and their scaling can not be determined by counting a suitable dimensionful parameter. In the threshold expansion this is reflected by the fact that we had to divide the small momentum region further into potential, soft and ultrasoft (see (田)) in order to arrive at a homogeneous expansion with manifest power counting.

Recently the possibility to factorize the small-momentum scales $m v$ and $m v^{2}(\sqrt{y}$ and $y / q$ in our notation) has been investigated in a series of papers [18, 19, 20,21,22]. Different rescalings were proposed in 19,20 that succeed in making power counting manifest in one region, but fail in others. Thus while the rescaling of [19] is adequate for potential gluons $\left(k_{0} \sim m v^{2}, \vec{k} \sim m v\right)$, the rescaling of 20 is tuned for ultrasoft gluons $\left(k_{0} \sim \vec{k} \sim m v^{2}\right)$ and the multipole expansion. Both rescalings are unified in the treatment of [21], where different fields for (non-propagating, off-shell) potential gluons and (propagating) ultrasoft gluons are introduced. At least for diagrams, in which only one gluon (massless) line is present, this approach appears to be equivalent to ours, as can be seen by comparing the treatment of the small momentum contributions to the triangle graph in Sect. 2 with the discussion in [21]. Furthermore, in the third line of Fig. 2 the first graph corresponds to the exchange of a potential gluon and the second to the exchange of a propagating gluon in the language of [21]. On the other hand, the existence of soft gluons, i.e. propagating gluons with energy and momentum of order $m v$, has not been considered in [19,20,21] and does not seem to fit into the rescaling schemes proposed there. Yet another approach has been taken in [22], where a non-local effective Lagrangian is proposed that follows after integrating out soft gluons. (In practice, this has been done only for single gluon exchange, which gives rise to the standard Coulomb 
potential with relativistic corrections. The discussion of the soft box subgraph in Sects. 2 and 4 suggests that the non-local Lagrangian can be extended to include the box graph contribution.) However, it has not yet been shown how to treat potential gluons in the approach of [22].

Labelle has first discussed the separation of soft/potential and ultrasoft photons and the multipole expansion in the context of NRQED [18]. It seems to us that Labelle's treatment of NRQED graphs is most closely related to our treatment of the small momentum regions in the threshold expansion. Technical differences arise in as much as he implies a cut-off regularization, while the simplicity of the threshold expansion discussed here is specific to dimensional regularization. In addition, 18 starts with time-ordered perturbation theory and Coulomb gauge, while we emphasize that the power counting and approximations in a given region can be carried out before performing the integration over the zero-components of the loop momenta and the method works in any gauge. (Since we have considered scalar propagators only, the question of gauge does not arise. In a gauge theory Coulomb gauge is very convenient for the threshold expansion, because propagating gluons which cause most complications couple to quarks through vertices that are suppressed by at least a factor of $\sqrt{y}$.) In this paper, as in [18 and in contrast to [19,20,21,22, no attempt is made to write down an effective Lagrangian after integrating out soft or soft together with potential gluons. This may be considered as a drawback. On the other hand, as this Lagrangian would inevitably be non-local, its utility is not obvious to us, once manifest power counting can be achieved otherwise.

After this conceptual postludium we should emphasize that the main focus of this paper is on the ability to perform calculations. The threshold expansion method described here allows us to treat 2-loop 3-point functions and opens the door to threshold problems at next-to-next-to-leading order. A first realistic application will be treated in a companion paper [15].

Acknowledgements. We thank A. Czarnecki, A. Davydychev and K. Melnikov for helpful discussions and providing checks on some of the integrals required for the hardhard region. We are also grateful to A.V. Kotikov and I.Z. Rothstein for useful discussions and to W. Beenakker and B. Tausk for correspondence regarding the exact box graph. V.S. thanks the CERN Theory Group and the Universities of Leiden and Mainz for hospitality during the course of this work. Both, V.S. and M.B. thank the Theory Group at the University of Karlsruhe for hospitality while this work was completed. Thanks to G. Buchalla and K. Chetyrkin for reading the manuscript. The work of V.S. has been supported by the Russian Foundation for Basic Research, project 96-01-00726, and by INTAS, grant 93-0744. 


\section{References}

[1] S.G. Gorishny, Nucl. Phys. B319 (1989) 633.

[2] K.G. Chetyrkin, Teor. Mat. Fiz. 75 (1988) 26; ibid. 76 (1988) 207; Max-PlanckInstitute preprint MPI-PAE/PTh 13/91 (Munich, 1991).

[3] V.A. Smirnov, Commun. Math. Phys. 134 (1990) 109; V.A. Smirnov, Renormalization and asymptotic expansions (Birkhäuser, Basel, 1991).

[4] V.A. Smirnov, Mod. Phys. Lett. A10 (1995) 1485; Talk given at 5th International Conference on Physics Beyond the Standard Model, Balholm, Norway, 29 Apr - 4 May 1997 hep-ph/9708423.

[5] F.V. Tkachov, INR-F5T-97-01 [hep-ph/9703424].

[6] K.G. Chetyrkin and V.A. Smirnov, Phys. Lett. 144B (1984) 419.

[7] D.J. Broadhurst, J. Fleischer and O.V. Tarasov, Z. Phys. C60 (1993) 287; A.I. Davydychev, V.A. Smirnov and J.B. Tausk, Nucl. Phys. B410 (1993) 325; F.A. Berends, A.I. Davydychev, V.A. Smirnov and J.B. Tausk, Nucl. Phys. B439 (1995) 536; F.A. Berends, A.I. Davydychev and V.A. Smirnov, Nucl. Phys. B478 (1996) 59.

[8] N. Gray, D.J. Broadhurst, W. Grafe and K. Schilcher, Z. Phys. C48 (1990) 673.

[9] K.G. Chetyrkin and F.V. Tkachov, Nucl. Phys. B192 (1981) 159.

[10] O.V. Tarasov, DESY-96-240 hep-ph/9703319].

[11] A. Djouadi, Nuovo Cim. 100A (1988) 357; D.J. Broadhurst, Z. Phys. C47 (1990) 115.

[12] W. Beenakker and A. Denner, Nucl. Phys. B338 (1990) 349.

[13] W.E. Caswell and G.P. Lepage, Phys. Lett. B167 (1986) 437; B.A. Thacker and G.P. Lepage, Phys. Rev. D43 (1991) 196; G.P. Lepage, L. Magnea, U. Magnea, C. Nakleh and K. Hornbostel, Phys. Rev. D46 (1992) 4052.

[14] V.A. Smirnov, Phys. Lett. B394 (1997) 205; A. Czarnecki and V.A. Smirnov, ibid. B394 (1997) 211; V.A. Smirnov, Phys. Lett. B404 (1997) 101.

[15] M. Beneke, A. Signer and V.A. Smirnov, in preparation.

[16] J. Fleischer and O.V. Tarasov, Comp. Phys. Commun. 71 (1992) 193.

[17] K.G. Chetyrkin, J.H. Kühn and M. Steinhauser, MPI-PHT-97-029 hep$\mathrm{ph} / 9705254$.

[18] P. Labelle, MCGILL-96-33 hep-ph/9608491. 
[19] M. Luke and A.V. Manohar, Phys. Rev. D55 (1997) 4129.

[20] B. Grinstein and I.Z. Rothstein, UCSD-97-06 hep-ph/9703298.

[21] M. Luke and M.J. Savage, UTPT-97-12 hep-ph/9707313, to be published in Phys. Rev. D56.

[22] A. Pineda and J. Soto, UB-ECM-PF-97-17 hep-ph/9707481, to be published in the proceedings of High-Energy Physics International Euroconference on Quantum Chromodynamics: QCD 97, Montpellier, France, 3-9 Jul 1997. 